\title{
Proteomics and the inner ear
}

\author{
Isolde Thalmann* \\ Washington University School of Medicine, \\ Department of Otolaryngology, St. Louis, MO 63110, \\ USA
}

The inner ear, one of the most complex organs, contains within its bony shell three sensory systems, the evolutionary oldest gravity receptor system, the three semicircular canals for the detection of angular acceleration, and the auditory system - unrivaled in sensitivity and frequency discrimination. All three systems are susceptible to a host of afflictions affecting the quality of life for all of us.

In the first part of this review we present an introduction to the milestones of inner ear research to pave the way for understanding the complexities of a proteomics approach to the ear. Minute sensory structures, surrounded by large fluid spaces and a hard bony shell, pose extreme challenges to the ear researcher. In spite of these obstacles, a powerful preparatory technique was developed, whereby precisely defined microscopic tissue elements can be isolated and analyzed, while maintaining the biochemical state representative of the in vivo conditions.

The second part consists of a discussion of proteomics as a tool in the elucidation of basic and pathologic mechanisms, diagnosis of disease, as well as treatment. Examples are the organ of Corti proteins OCP1 and OCP2, oncomodulin, a highly specific calcium-binding protein, and several disease entities, Meniere's disease, benign paroxysmal positional vertigo, and perilymphatic fistula.

Keywords: Cochlea, organ of Corti, gap junctions, proteomics, oncomodulin, Meniere's disease, benign paroxysmal positional vertigo, otoconia, perilymphatic fistula

\section{Introduction to the ear}

The inner ear, one of the most complex organs, contains within its bony shell three sensory systems, the evolutionarily oldest gravity receptors, the three semi-

* Address for correspondence: Isolde Thalmann, Ph.D., Washington University School of Medicine, Department of Otolaryngology, 660 S. Euclid Avenue, St. Louis, MO 63110, USA. Tel.: +1 314 362 7505; Fax: +1 314362 7568; E-mail: thalmanni@msnotes. wustl.edu. circular canals for the detection of angular acceleration, and within the same cavity connected by the endolymphatic fluid system, the phylogenetically far younger cochlea, the organ of hearing (Fig. 1(A)). The essential element of these microscopic sensory organs are the hair cells, specialized mechanoreceptors, which are in contact with endolymph, a fluid with highly unusual ionic composition (Figs 1(B)-(D)).

It is obviously an enormous undertaking to cover in a short review how this highly specialized organ accomplishes its tasks, how it can be studied from a proteomics standpoint, what is known to go wrong, and where we stand on fixing problems. A didactic approach is used, frequently requiring oversimplification. Nevertheless, we try to present a realistic state-of-theart assessment of the system in terms of its individual components, and at an integrated level. The focus is on the auditory system with its highly specialized receptor, the organ of the Corti. However, due attention is given to two indispensable support systems: 1 . the endolymphatic sac, the main regulatory center that plays a key role in Meniere's disease, and 2. the gravity receptor with its mineral particles that provide its exceptional sensitivity.

Some 30 million Americans suffer from hearing impairment; one of every 1000 infants is born deaf and many more individuals are afflicted by genetic disorders resulting in deafness later in life. An even greater number at some time experience problems of balance or dizziness. In fact, a certain degree of hearing impairment, and degenerative processes in the balance organs, are normal features of aging and affect our quality of life. Care for these disorders exceeds a billion per year.

The role proteomics has played in basic and clinical inner ear research is discussed, as well as the challenges of the future. For each project the primary approach, discovery vs hypothesis-driven, global vs specific, is stated. Disease entities covered as examples are Meniere's disease, benign paroxysmal positional vertigo (BPPV), perilymphatic fistula, and non-syndromic genetic hearing loss.

\section{Milestones in inner ear research}

The modern era of inner ear research was launched with the discovery of the organ of Corti around 1850 [1]. 


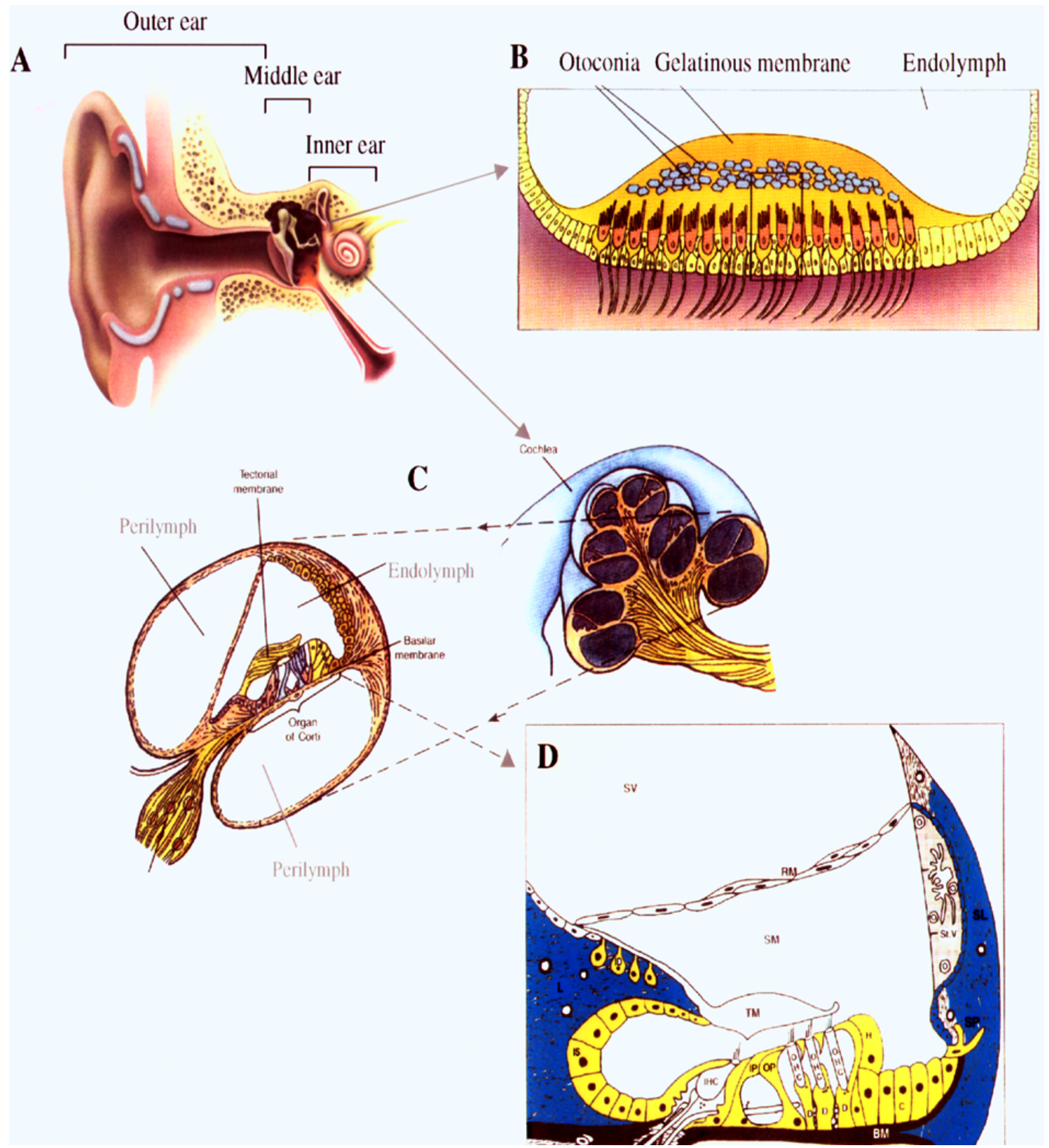

Fig. 1. (A) Semidiagrammatic drawing of the ear with the temporal bone cut away to reveal the inner ear consisting of semicircular canals, vestibule and cochlea. (B) Drawing of the cross-section of the gravity receptor of the saccule showing the hair cells, supporting cells and the sensory superstructure, composed of the gelatinous membrane with the embedded otoconia. (Adapted from [42].) (C) (RIGHT) Drawing of a midmodiolar section through a human cochlea showing the three turns. Note the huge fluid spaces and the modiolus in the center which contains the fibers of the auditory nerve. The enlarged cross section (LEFT) shows the three fluid spaces of the cochlea, endolymph of scala media, and the two perilymphatic compartments. The organ of Corti, the auditory receptor, rests on the basilar membrane and is covered by the tectorial membrane. The stria vascularis, the main transport organ, is located on the lateral wall of the cochlear duct, and is backed by the spiral ligament. (Adapted from [43].) (D) A higher power cross-section through the cochlear duct showing details of the organ of Corti, and the stria vascularis (StV). The supporting cells of the organ of Corti and adjacent nonsensory cells are connected by gap junctions, constituting the epithelial gap junction system (yellow). The spiral ligament and spiral limbus comprise the connective tissue gap junction system (blue). The two gap junction systems interact and are believed to be responsible for the removal of $\mathrm{K}^{+}$emanating from the hair cells. IHC - inner hair cells; OHC - outer hair cells; IP - inner pillar cells; OP - outer pillar cells; D - Deiters' cells; H - Hensen cells; C - Claudius' cells; IS - inner sulcus; ID - interdental cells; L - limbus; SP - spiral prominence; SL - spiral ligament; RM - Reissner's membrane; BM - basilar membrane; SV - scala vestibuli; ST scala tympani; SM - scala media. (Adapted from [44].) 
The first electrophysiological studies in the 1920s led to the discovery of the cochlear microphonics, the auditory receptor potential, which is distinct from the action potential $[2,3]$. In contrast to the all or nothing response of the action potential, cochlear microphonics are essentially electrical analogs of the acoustical stimulus. Von Bekesy inferred that the transduced electrical signal contained significantly more energy than the acoustic stimulus. In search for the required amplifier he discovered the unprecedented positive potential $(+80 \mathrm{mV})$ of the endolymphatic space, the endolymphatic potential [4]. This inspired the first targeted quantitative chemical study by Smith, Lowry and $\mathrm{Wu}$ who discovered the equally unprecedented ionic profile of endolymph, a reversed $\mathrm{K} / \mathrm{Na}$ ratio with $\mathrm{K}$ being $150 \mathrm{mEq}$ and $\mathrm{Na} 1 \mathrm{mEq}$ [5]. The stria vascularis, as the name implies, a highly vascularized epithelial strip on the lateral boundary of the endolymphatic compartment (Fig. 1(D)), was tentatively implicated as the source of the electrochemical gradient and the generator of the endolymphatic potential. With the objective of localizing and characterizing the cochlear energy sources, a search for the metabolic characteristics of the structures in question was initiated. Matschinsky and Thalmann adapted the powerful analytical techniques developed by Lowry for studies of the brain [6], to the unusual anatomical features of the ear [7] incorporating: 1. An analytical technique of the necessary sensitivity and specificity, and 2 . a preparatory technique satisfying two essential requirements: a. maintenance of the in vivo metabolic state, achieved by rapid freezing and freeze-drying. b. spatial resolution by dissection of microscopic tissue elements at a level dictated by the aims of the study. This preparatory scheme, originally used in energy studies, has withstood the test of time and has served unchanged as basis for high resolution proteomics to follow much later.

Using the behavior of labile metabolites as indicators of the energy status, together with information from a simpler model system, the stria vascularis was confirmed as the cochlear power plant, the source of the $\mathrm{K}$ current that flows through the hair cells of the organ of Corti $[8,9]$. The stereocilia of the hair cells modulate the current flow by sound-synchronous deflections, thereby modulating the current flow and setting up the receptor potential.

This essentially was the picture some 25 years ago. However, the situation was soon to be shaken up when Spoendlin found that only the inner hair cells are innervated by afferent fibers and that the outer hair cells are in essence connected only to efferent input [10]. This defined the inner hair cells as the only true auditory receptor cells, cells incorporating all three requirements, the sensing, the transduction, and the transmission elements; it left the glamorous, highly vulnerable outer hair cells out in left field without an obvious role. However, shortly thereafter a series of discoveries uncovered an entirely unanticipated level of refinement of the auditory receptor, superimposed upon the traditional model formulated in the $70 \mathrm{~s}$.

The following are the key developments:

1. In 1978 Kemp discovered the spontaneous and evoked acoustic emissions, which could not be explained by the classical model but revealed an active process in the cochlea [11].

2. Brownell et al. in 1985 discovered the electrically induced motility of the outer hair cells [12].

3. Finally, Zheng et al. (2000) identified and characterized the motor protein prestin in the lateral plasma membrane of the outer hair cells [13].

The picture as we see it today in a nutshell: The inner hair cells are the only cells able to "hear" in the true sense, albeit at a rather rudimentary level. The outer hair cells could, in a sense, be considered 'disabled' receptor cells because they, even though equipped with the sensing and transducer elements, are unable to trigger release of afferent transmitter. Instead, the receptor potential is converted by a process of reverse transduction into synchronous change in the length of the hair cells. In essence the minute acoustic (micromechanical) signals are converted back into mechanical energy, but on a far higher level. Ultimately the motions of the outer hair cells provide the inner hair cells with a vastly amplified mechanical input. This synergism of the outer hair cells with the inner hair cells is the basis of the superb performance of the mammalian auditory receptor which is characterized by high sensitivity and frequency selectivity, in combination with an immense dynamic range.

The function of the efferent system is highly complex, but adjustment of the gain of the motor is one essential aspect. In the absence of outer hair cells, the inner hair cells are still able to perceive and transmit sound but at a far reduced level of sensitivity and selectivity. (For a comprehensive review see Dallos et al. [14].)

It should be obvious that a great number of steps, predominately mediated by proteins, go on simultaneously within this tiniest of places. It must be evident even to the most enthusiastic proponent of the universal power of DNA technology, that the fine workings of this machinery can only be unraveled by means of a highly selective proteomics approach. 


\section{Proteomics of the ear}

\subsection{Global vs. specific approach}

Proteomics is at best a daunting project. A choice must be made whether to use a global approach, i.e. trying to take inventory of proteins in various structures - of necessity with rather diffuse goals - or rather focus on subsets of protein(s) with challenging biological or medical properties. As we shall see, the latter approach, derogatorily called the "cottage industry approach" - and claimed to be the principal strategy used by academia - proves to be the preferred one. In the case of the ear the choice of approach is dictated by two factors: 1. anatomical features of the inner ear ; and 2. high-throughput tools. The high-throughput technology is not quite advanced enough to apply it on a large scale to the ear. A hard bony shell, and by comparison huge fluid spaces surrounding the fragile organ of Corti (an epithelial structure devoid of connective tissue and of vascularization), pose extreme challenges to the ear researcher. Moreover, the organ of Corti is postmitotic, and therefore one of the most important tools of proteomics, the use of cell lines, is precluded. The problems are compounded by additional constraints when dealing with the human ear and with pathobiochemical events underlying the various disease entities. The examples to be described will indicate that an eclectic approach which combines both, global and specific approaches, is ultimately most profitable.

\subsection{Importance of preparatory technique}

The availability of a powerful micropreparatory technique for inner ear tissues and fluids developed earlier for metabolic studies (see Introduction) proved to be a major asset for the proteomics application. The importance of a representative sample cannot be sufficiently emphasized. For instance, measurements based on the analysis of the whole cochlea, are entirely useless. The data contributed by the miniscule sensory structures of interest - each with its specialized role - are entirely obscured by the huge amounts of bone, connective tissue and the fluids. To briefly reiterate, the technique, originally developed for metabolic studies, faithfully maintains the existing metabolic state and allows correlation of the biochemistry of precisely defined microscopic tissues elements with the underlying electrophysiologically controlled functional parameters $[15,16]$. This is accomplished by rapid freezing, freeze-drying in toto (which preserves the three-dimensional structure of in- ner ear tissues), and dissection of substructures of the organ of Corti in the freeze-dried state (if required to the level of single outer hair cells). A windfall of this technique is that, because the tissue is freeze-dried, no interferences are introduced with, buffers, salts, contaminants from extraction procedures, and there are no concentration problems. Lysis buffer can be added directly prior to 2D-PAGE, for instance.

\subsection{Examples of the application of proteomics to basic and clinical inner ear research}

\subsubsection{Organ of Corti proteins (OCP1 and OCP2)}

Shortly after 2D-PAGE became available in the late $70 \mathrm{~s}$, we applied it to the structure of greatest interest, the organ of Corti; we used the technique in a typical proteomics aim, the global discovery mode. We opted not to use the time-honored hypothesis-driven approach, but instead went on a "fishing expedition". In view of the limited sensitivity and a modest sample size (30 ug of total protein of organ of Corti from the ears of two guinea pig), we expected at best to detect the most prominent structural proteins, such as tubulin and actin, and some of the more abundant housekeeping proteins. Instead, as seen in Fig. 2 (left) the two most prominent spots corresponded to two unknown, low molecular weight, acidic proteins. Because they were not detectable in other tissues with 80's technology, we termed them OCP1 and OCP2 [17]. Jumping ahead 20 years, we now know that we had discovered in a most unlikely location, i.e. in the remote organ of Corti, two proteins of universal importance. OCP2 turned out to be a close homolog of SKP1, a protein involved in control of the cell cycle, originally characterized in yeast [18], and OCP1 the first representative of the family of F-box proteins [19]. We hypothesize, that together with cullin-1 OCP1 and OCP2 form the so called SCF complex which targets specific proteins for ubiquitination and proteolysis by the proteasome (Fig. 3). Typical targets of the SCF complex in other systems are cell cycle proteins, such as cyclins and their inhibitors. However, a role of OCP1 and OCP2 in cell cycle control in the organ of Corti can be ruled out because of:

1. the abundance of the proteins (5\% of total protein);

2. the cells of the organ of Corti are postmitotic;

3. their location in the supporting cells rather than the hair cells, specifically within the cells of the epithelial gap junction system $[21,22]$. 
A

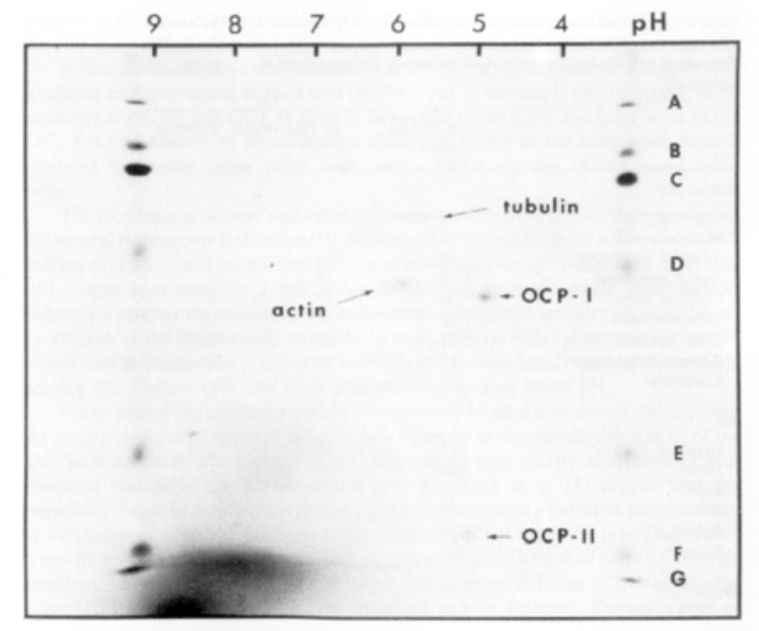

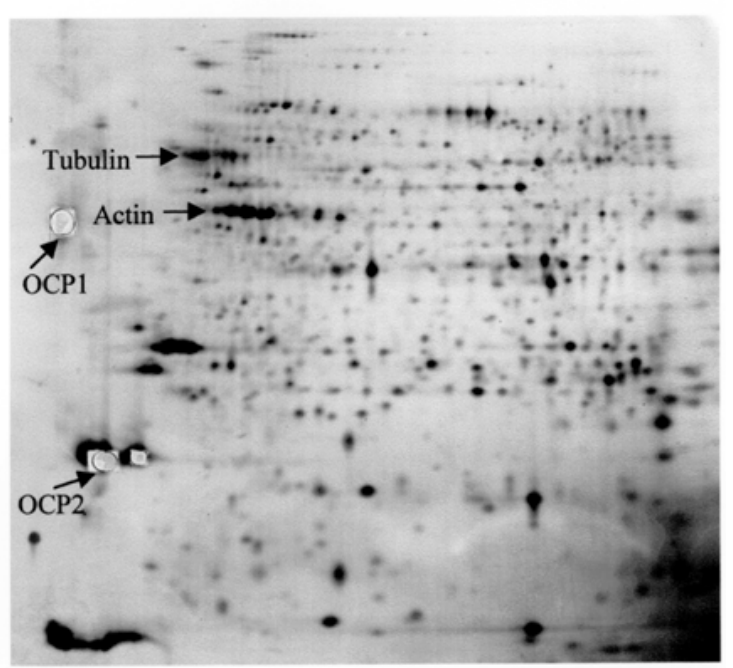

Fig. 2. Two-dimensional separation of polypeptides from organ of Corti of guinea pig obtained (A) with 1980's technology (reproduced from [17], with permission) and (B) today's technology. For the gel on the right IPG (pH 3-10L) was used in the first dimension, ISO-DALT (9-18\% acrylamide gradient) in the second dimension. $30 \mathrm{ug}$ and $50 \mathrm{ug}$ of total protein were loaded onto gels A and B, respectively. OCP1 and OCP2 were excised from gel B for amino acid sequencing. Because of early conventions, the left gel represents a mirror image. Gel A was Coomassie stained only, gel B was Coomassie stained, followed by silver staining. (For details of method see our Washington University Inner Ear Protein Database http://oto.wustl.edu/thc/innerear2d.htm.)

The target protein of the SCF complex in the organ of Corti remains conjectural, but indirect evidence points to connexin 26 and/or 30 because: 1 . of the exact colocalization of the OCPs with connexin 26 and 30; 2 . connexins are the only substrates expressed at comparative levels; 3 . most connexins are known to turn over rapidly, and are controlled by proteolytic degradation in some systems, e.g. the heart [23]. A rapid turnover has recently been documented also for the cochlear gap junctions [24]. Figure 3 shows a model of the hypothetical role of the OCP1/OCP2 complex in connexin ubiquitination. (For details see [22].)

Upshot: The two proteins, OCP1 and OCP2, together with cullin-1, form a regulatory complex that is believed to control an essential service system of the cochlea, the gap junction system. This syncytium of support cells aids in the removal of toxic waste, specifically the large amounts of $\mathrm{K}$ emanating from the hair cells, thereby counteracting the biological hardships imposed by a hostile electrochemical environment. Moreover, this system undoubtedly also serves for metabolic equilibration and electrical synchronization in analogy to the syncytium of the myocardium [23]. The importance of this service system is evident from the fact that: a) mutations in connexin 26 and 30 represent the predominant pathogenetic substrate of nonsyndromic hearing loss (over 50\%); b) the OCP1 gene is located on the short arm of chromosome 1 in a region that has been implicated in nonsyndromic hearing loss [25].

\subsubsection{Oncomodulin (OM)}

Detection of OM in the organ of Corti occurred by chance during the course of a targeted study aimed at confirmation of a potential calcium-binding function of OCP2 [26,27]. The experimental set up in this case involved in effect a global search directed at the subset of calcium-binding protein of the organ of Corti. Subsequently we determined that the expression of OM is limited to the outer hair cells and that the protein accounts for as much as $0.5 \%$ of the total $[28,29]$.

Also the original discovery of OM was due to chance during the screening of human tumor cell lines [30]. This accounts for the 'onco' portion of the name, while the "modulin" portion derives from certain similarities with calmodulin. This combination of features spurred vigorous interest in cancer research circles. However, the search for a physiological expression site was largely unsuccessful since it yielded only one low expression site in preimplantation embryonic tissue. Consequently prior to discovery of cochlear expression, OM was considered an "onco-developmental" protein. This is a graphic example of the futility of a strictly global search since it cannot possibly detect expression in an isolated microscopic structure, sequestered within a protective bony shell. 

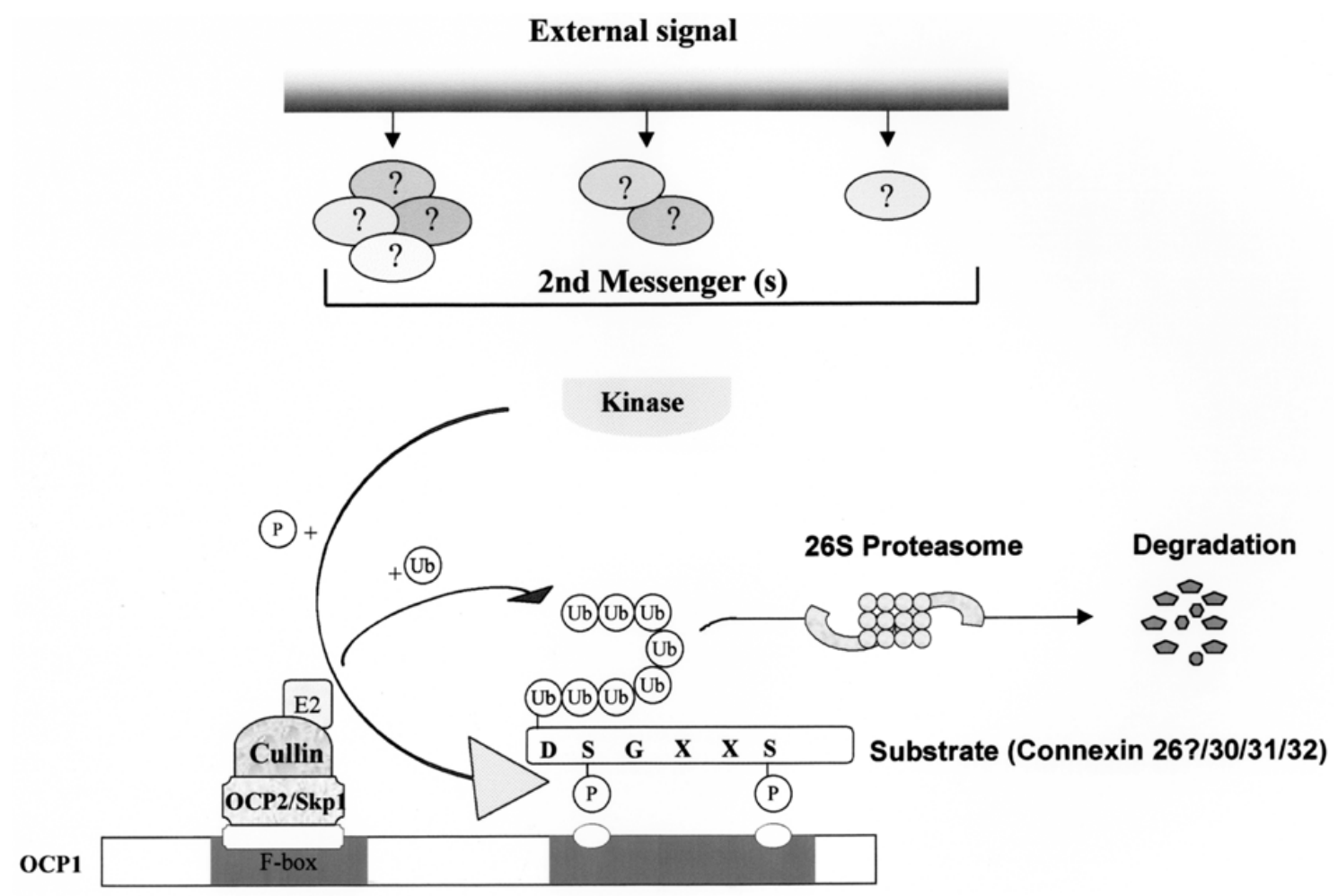

Fig. 3. Model of the function of the SCF in ubiquitination. External signals activate the corresponding kinase, which modifies the substrate connexin at the phosphorylation site (S). The modified substrate links the target protein to the SCF-E2 ubiquitination apparatus, leading to the formation of a multiple ubiquitin chain. The multiubiquitinated protein is subject to the proteolysis by the $26 \mathrm{~S}$ proteasome. This model could explain the high turnover of connexin. We speculate a similar mechanism for the putative OCP1/OCP2/cullin complex which could play an important role in the epithelial gap junction system. (See text for details.) (Adapted from [45].)

The precise function of OM remains uncertain. However, the high level of expression restricted to the outer hair cells implies involvement in the specialized function of this unique cell type. This could either concern involvement in the motor process per se, or a role in the modulation of the gain of the motor by the efferent system. A direct involvement in the operation of the motor per se is not a viable option, because of its extremely rapid time scale, which can only be accounted for by electrically induced conformational changes. The most obvious alternative, therefore, would be involvement in the modulation of the gain of motor. An attractive hypothesis would postulate a role of OM in the mediation of the "slow efferent" cholinergic effect which modifies the gain of the motor in a calcium-dependent manner [29,31].

\subsubsection{Meniere's disease}

The detection of the OCPs is an example of a discovery-driven, global approach, while that of OM was a chance observation during a targeted study based on a global strategy of limited scope. The following example shows another version of a discovery-driven approach with a specific target in mind, the subset of glycosylated proteins of endolymph. An estimated 1/2 to 1 million people in the United States suffer from Meniere's disease. The symptoms include attacks of vertigo, fluctuant hearing loss, tinnitus, and fullness.

Figure 4(A) shows a cross-section of a cochlea of a patient with this disease, showing severe hydrops, i.e. increase in endolymph volume. Compelling experimental evidence implicates the endolymphatic sac as the center for volume regulation of the endolymphatic compartment [32] (Fig. 4(B)). An electrophysiologically controlled fluid dynamic study, combined with histochemical evaluation of the endolymphatic sac, showed that the 'homogeneous substance' that normally occupies the sac lumen, rapidly disappears in response to expansion of endolymph volume by injection of artificial endolymph, while the opposite is true when the volume is contracted (Fig. 4(C)-(D)) [33]. The 'homogeneous substance' appears to be a composite of 
A
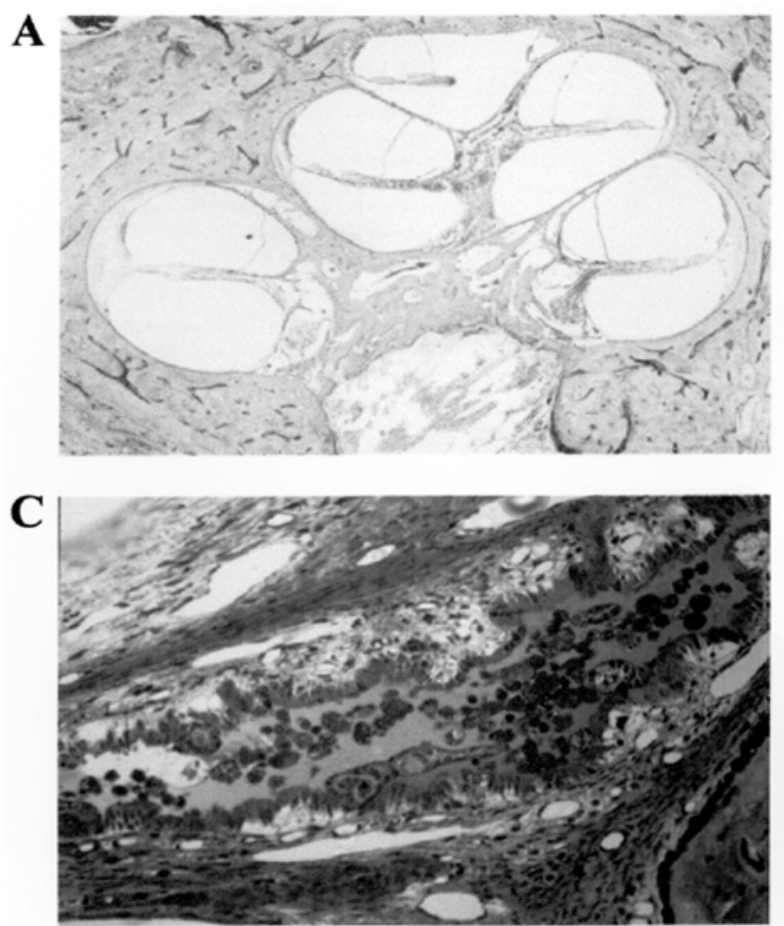

Semicircular duct:
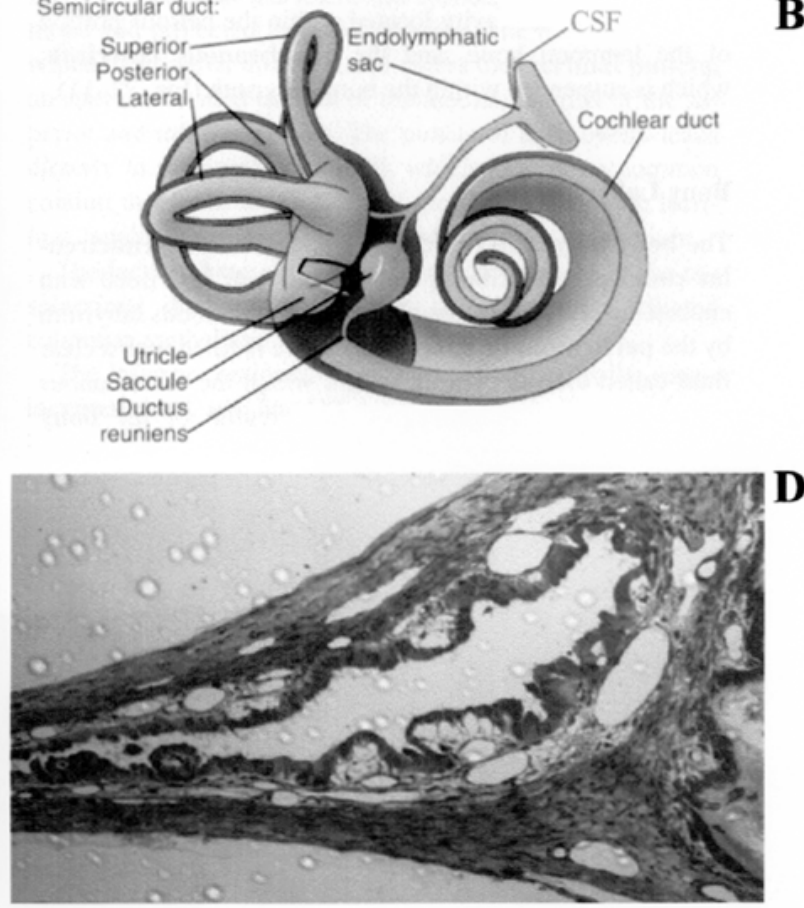

Fig. 4. (A) Photomicropgraph of a cross-section of the right cochlea of a patient, showing severe cochlear hydrops. Note the enormous distention of the endolymphatic space which displaces perilymph of scala tympani. For comparison see the normal situation in Fig. 1. (From [46], with permission.) (B) Schematic drawing of the inner ear (see Fig. 1(A)) with parts of the membranous labyrinth exposed, including cochlear duct and endolymphatic sac. The endolymphatic sac is the structure controlling endolymph volume. (C, D) Light micrograph of a cross-section through the endolymphatic sac of the guinea pig. (C) control; (D) experimentally induced expansion of the endolymphatic space by injection of endolymph (injection time $7.5 \mathrm{~min}$ ). Note almost total absence of the normal intraluminal 'homogeneous substance' on the injected side when compared with the control ear. (From [33], with permission.)

glycosylated macromolecule(s). Our objective was to identify the chemical nature of this substance(s). By treating the fluid content of the endolymphatic sac with various deglycosylating enzymes prior to 2D-PAGE, we arrived at the subset of proteins that is presumed to include the 'homogeneous substance' (Fig. 5) [34].

In this project the main difficulty was procurement of uncontaminated fluid samples. Not only is the endolymphatic sac exceedingly small, holding about $200 \mathrm{nl}$ compared to $4 \mathrm{ul}$ of endolymph and some $30 \mathrm{ul}$ of perilymph (in guinea pig), but its wall consists of a highly vascularized soft tissue layer. Therefore liquid sampling routinely used for cochlear endolymph which is encased in the bony shell, cannot be used effectively in the sac. We developed a technique whereby the content of the endolymphatic sac can be collected in its entirety in the freeze-dried state, free of contaminants.

The most fundamental difference between the endolymphatic sac and peripheral endolymph is the level of total protein which is 50 fold higher in the sac. However, there are also several marked differences in the profile of the proteins (Fig. 5). We determined that midmolecular weight, acidic proteins, make up the majority of detectable proteins, and that most of these are degraded by deglycosylating enzymes. One or several of these proteins presumably correspond(s) to the histologically visible 'homogeneous substance'. At least two levels of reference need to be considered when evaluating the profiles of the fluid of the sac: 1. Comparison of the profile of sac endolymph with that of peripheral endolymph residing within the same compartment, and comparison with the profiles in contiguous compartments, serum, perilymph and CSF; 2 . experimental manipulation of endolymph volume, which results in a corresponding increase and decrease in the density of the histologically visible 'homogeneous substance'. Using these approaches in combination, several proteins have been selected as candidates, and tentatively identified on the basis of their electrophoretic mobility; others are being processed for partial amino acid sequencing and/or mass spectrometry. 

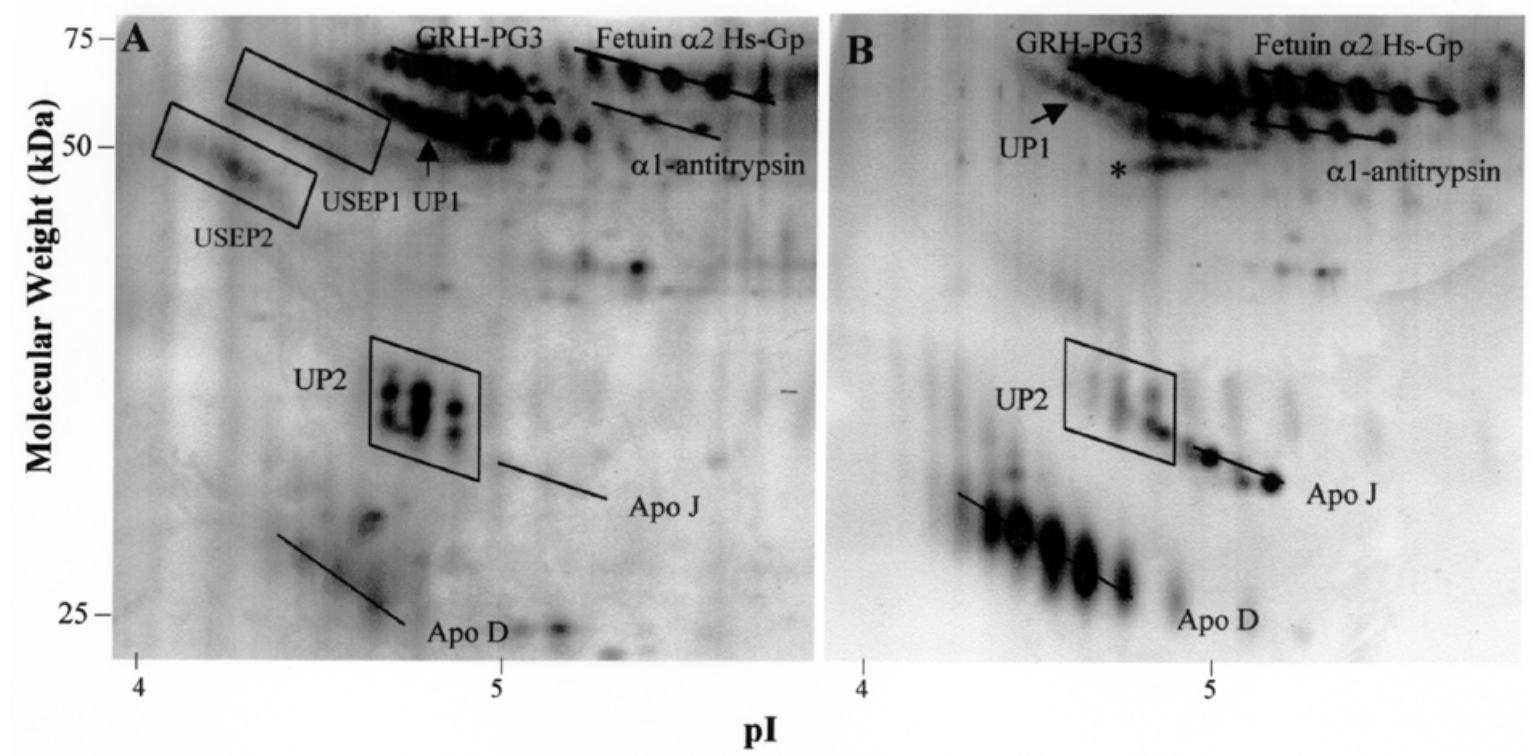

Fig. 5. A portion of a two-dimensional separation of guinea pig endolymph derived from: A. endolymphatic sac, and B. cochlea. Sample volumes were adjusted for equal total protein content to facilitate estimation of relative abundance of corresponding spots. Note the two relatively abundant spots, UP1 and UP2 (unidentified protein), and two that we termed USEP1 and 2 (unidentified sac protein) because we could not detect them in the cochlear endolymph. Apolipoproteins D and J (apo D and apo J) show exceptionally high levels in cochlear endolymph. They are known to be present also in high concentrations in CSF.

The currently most effective treatment for relief of the symptoms of the incapacitating vertigo of severe Meniere's disease is destruction of the vestibular sensory organs by judicious use of ototoxic drugs, attempting to spare the cochlea. By identifying the substance(s) that control(s) endolymph volume, we hope to lay the basis for a rational therapeutic scheme aimed at correcting the dysfunction of volume regulation in a targeted, nondestructive way.

\subsubsection{Non-syndromic hearing impairment}

Most genetic hearing impairments are non-syndromic (hearing impairment without other clinical abnormalities). In most instances it was not the discovery of a protein that led directly to identification of the gene(s) responsible for the deafness (except for OCP1). Therefore we refer the reader to a database for more information [35].

\subsubsection{Otoconia and benign paroxysmal, positional vertigo $(B P P V)$}

The gravity receptor organ contains a gelatinous sensory superstructure into which biomineral particles, called otoconia, are embedded for enhancement of gravity perception (Fig. 1(B)). Morphogenesis of otoconia is a highly controversial issue, and it is not known whether otoconia once formed during the embryonic stage persist throughout the entire life span, or whether they can be repaired or regenerated following damage. It is firmly established that human otoconia of the saccular portion of the vestibule tend to degenerate starting at age 50-60 and this undoubtedly contributes to the instability and dysequilibrium experienced by the majority of the elderly. Recent interest in the pathobiology of otoconia surged following the realization that otoconial pathology is causally involved in one of the most prevalent otoneurologic entities BPPV which preferentially affects younger age groups [36].

Apart from the mechanisms involving morphogenesis, one of the clinically most important questions is whether and to what extent the organic matrix is involved in the maintenance and the demise of otoconia. Highly pertinent in this context is the purported ability of otoconia to regenerate following destruction by ototoxic drugs [37]. As a first step in our attempts to identify the factors responsible for maintenance, repair and regeneration of otoconia, and of devising remedies to prevent degeneration or promote repair, we set out to characterize the otoconial matrix proteins. Briefly, matrix proteins were extracted from decalcified otoconia, separated by 2D-PAGE and the main matrix protein otoconin 90 partially sequenced and cloned [38, 39]. The molecule in question turned out to be a close homolog of phospholipase A2, evidently drafted for its 
role in the organization of a mineral structure for its rigidity imparted by seven pairs of disulfide bridges. The molecule was further adapted to its role in mineralization by numerous substitutions with acidic residues and incorporation of a massive endowment with complex polysaccharides. The subsequently obtained expression patterns of mRNA and protein in the embryonic ear were in conflict with traditional concepts and required revision of the theory of otoconial morphogenesis for the following reasons: 1 . Morphogenesis of otoconia is completed prior to onset of function; 2 . Contrary to traditional teachings, the protein is not synthesized and secreted in the sensory region, but in the adjacent nonsensory epithelia. This scheme is plausible from a teleological standpoint since it delegates the menial job of mass production of biomineral particles to less specialized areas of the gravity receptor organ, sparing the highly specialized sense organ. 3 . expression of the matrix proteins is not limited to the gravity receptor organ, but is plentiful throughout the entire membranous labyrinth. The question therefore arises why the formation and/or persistence of otoconia is restricted to the gravity receptor organ. This question has not been answered conclusively, but obviously an interaction of otoconin 90 with one or more factors localized to the gravity receptor must be postulated. The most promising candidate is the so-called matrix vesicles secreted by the supporting cells of the sensory region, which are held in place by the gelatinous phase of the gravity superstructure (see [39] for details). In this hypothetical model, interaction of otoconin 90 with acidic phospholipids of the matrix vesicles would initiate nucleation and growth of the calcium carbonate crystals in the low calcium environment of endolymph.

How can information about otoconin 90 and other minor matrix proteins help in the understanding of the biologic factors leading to degeneration and/or translocation of otoconia, and thereby clarify the pathogenesis of BPPV? 1. Ongoing experiments on the behavior of the otoconial matrix proteins and the regenerative events following streptomycin intoxication should provide insight into the processes governing the upregulation of embryonic otoconial genes, and thereby provide information how such processes could be induced biochemically without the need to injure the tissues with toxic agents. 2. Observation of the behavior of the biochemical properties of otoconin 90 of human otoconia prior to onset of degeneration should provide important insights into pathobiologic mechanisms occurring on an expanded time scale. The study of human material (obtained during the course of remedial surgery) is in- dispensable: 1. Because it allows comparisons of the behavior of matrix proteins with corresponding pathologic events; and 2. because of the enormous life span, during which wear and tear of inorganic components becomes a significant factor.

\subsection{Proteomics: A tool for diagnosis of diseases}

A limited global approach has been applied to the study of inner ear fluids that showed promise for the development of a diagnostic tool for specific inner ear disorders.

\subsubsection{Perilymphatic fistula}

In the mid 90s an inventory (global approach) of the proteins of the inner ear fluids of the guinea pig, and comparison with CSF and plasma resulted in the important findings that ultrafiltration cannot be the sole mechanism of perilymph production, and that endolymph is derived from perilymph rather than directly from blood [40]. The 2-dimensional electrophoretic protein profile indicated several significant differences from human perilymph obtained during surgery or post mortem. Most conspicuous were extremely high levels of apolipoprotein $\mathrm{J}$ and $\mathrm{D}$, and the presence of beta-2 transferrin, a protein absent in plasma, but also present in CSF [41] (Fig. 6).

It was attempted to use these proteins as markers for the diagnosis of perilymph fistula, one of the most controversial and challenging problems facing otologists. Perilymph fistula is an abnormal communication between the middle and inner ear, and the clinical signs include vertigo, tinnitus, aural fullness, and sensorineural hearing loss. In the search for markers several unanticipated problems arose which proved difficult to overcome: 1. Perilymph samples obtained during surgery frequently contained traces of plasma, and/or middle ear transudate, and 2. none of the proteins (except for beta-2 transferrin) are entirely specific for perilymph. Note that a small contamination of plasma in terms of volume, is a pronounced contamination in terms of concentration (total protein of plasma:perilymph is 30:1). Beta-2 transferrin, while in theory a highly specific marker for perilymph (and $\mathrm{CSF}$ ), was of little practical use in immunochemical studies, because commercially available antibodies recognized many other transferrin isoforms and masked the beta- 2 isoform. However, with the advent of ProteinChip techniques such as SELDI (Surface Enhanced Laser Desorption/Ionization) beta-2 transferrin could reestablish itself as a viable candidate. 


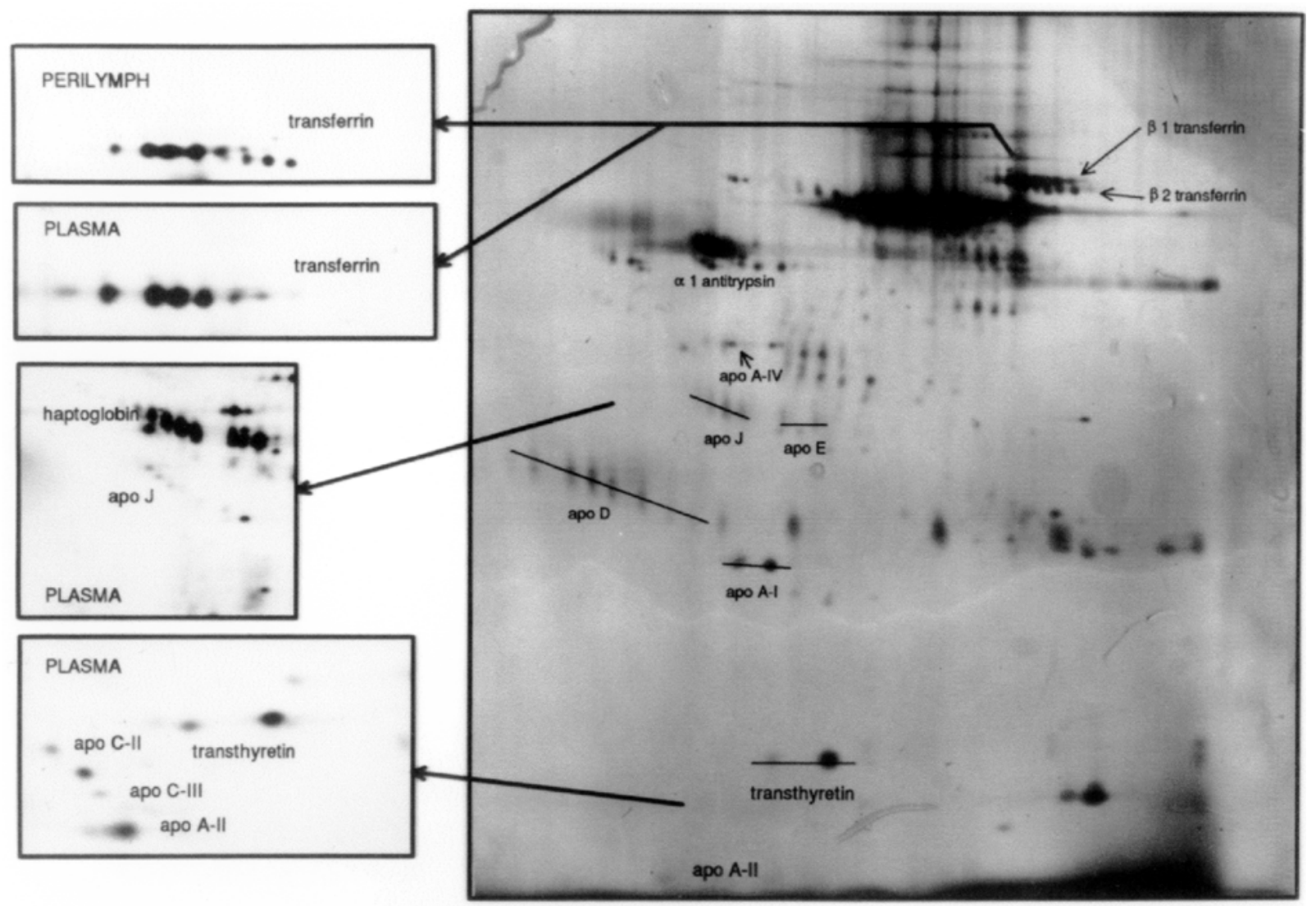

Fig. 6. Electrophoretic separation of proteins from perilymph of a 6.5 months old infant, collected 2.5 hours postmortem. 3 ul of perilymph were applied to the gel. The total protein content is approximately one-fourth of the adult level. Areas relevant to perilymph fistula diagnosis are enlarged on the left. (From [41], with permission.)

\subsubsection{Is the proteomics approach for diagnosis of other inner ear disorders meaningful?}

Abnormal protein profiles have been described for plasma/serum, cerebrospinal fluid, and urine (see other articles in this volume) for a variety of disorders. It is not envisioned that analysis of perilymph will be used for diagnostic purposes, but rather as an aid for the elucidation of the mechanisms underlying inner ear disease, whether localized or as part of systemic alterations. The major obstacle is procurement of inner ear fluid sample. Unlike plasma, urine, saliva, milk, sweat, and seminal fluid, an invasive and potentially dangerous procedure is involved. While procurement of CSF and amniotic fluid is invasive, the procedures are relatively straight forward and diagnostic benefits outweigh potential danger. A second obstacle is the minute volumes available, combined with their low protein levels. However, again, the new Protein Chip Technology theoretically is promising. However at this point, the technique does not warrant the immensely difficult and not risk-free sampling of inner ear fluids.

\section{Acknowledgments}

This work was supported by research grant $5 \mathrm{RO} 1$ DC01414 from the National Institute on Deafness and Other Communication Disorders, National Institutes of Health, and a grant from The National Organization for Hearing Research Foundation.

\section{References}

[1] A. Corti, Recherches sur l'organe de l'ouie des mammiferes premiere partie Lima con, Zeitschr $f$ wissenschaftl Zoology (1851), 3 .

[2] E.G. Wever and C.W. Bray, Action currents in the auditory nerve in response to acoustical stimulation, Proc Nat Acad Sci 16 (1930), 344-350. 
[3] S.S. Stevens and H. Davis, Hearing, Wiley \& Sons, New York, 1938.

[4] G. von Bekesy, Experiments in Hearing, McGraw-Hill, New York, 1960.

[5] C.A. Smith, O.H. Lowry and M.L. Wu, The electrolytes of the labyrinthine fluids, Laryngoscope 64 (1954), 141-153.

[6] O.H. Lowry, The quantitative histochemistry of the brain, Histological sampling, J Histochem Cytochem 1 (1953), 420-428.

[7] F.M. Matschinsky and R. Thalmann, Quantitative histochemistry of the organ of Corti, stria vascularis and macula sacculi of the guinea pig. I. Sampling procedure and analysis of pyridine nucleotides, Laryngoscope 66 (1967), 292-305.

[8] R. Thalmann, Metabolic features of auditory and vestibular system, Laryngoscope 81 (1971), 1245-1260.

[9] D.P. Corey and A.J. Hudspeth, Ionic basis of the receptor potential in a vertebrate hair cell, Nature 281 (1979), 675-677.

[10] H.H. Spoendlin, The innervation of the organ of Corti, J Laryngol 81 (1967), 717-738.

[11] D.T. Kemp, Stimulated acoustic emissions from within the human auditory system, J Acoust Soc Am 64 (1978), 13861391.

[12] W.E. Brownell, C.R. Bader, D. Bertrand and Y. de Ribaupierre, Evoked mechanical responses of isolated cochlear outer hair cells, Science 227 (1985), 194-196.

[13] J. Zheng, W. Shen, D.Z. He, K.B. Long, L.D. Madison and P. Dallos, Prestin is the motor protein of cochlear outer hair cells, Nature 405 (2000), 149-155.

[14] P. Dallos, A.N. Popper and R.R. Fay, eds, The cochlea, Springer, New York, 1996.

[15] R. Thalmann, Quantitative histo- and cytochemistry of the ear, in: The handbook of auditory and vestibular research methods, C.A. Smith and J.A. Vernon, eds, C.C. Thomas, Part III Biochemistry, Springfield, IL, 1976, pp. 359-419.

[16] R. Thalmann, I. Thalmann and T.H. Comegys, Quantitative cytochemistry of the organ of Corti. Dissection, weight determination and analysis of single outer hair cells, Laryngoscope 82 (1972), 2059-2078.

[17] I. Thalmann, H.L. Rosenthal, B.W. Moore AMD R. Thalmann, Organ of Corti-specific polypeptides: OCP-I and OCP-II, Arch Otorhinolaryngol 226 (1980), 123-128.

[18] C. Connelly and P. Hieter, Budding yeast SKP1 encodes an evolutionarily conserved kinetochore protein required for cell cycle progression, Cell 86 (1996), 275-285.

[19] C. Bai, P. Sen and K. Hofmann et al., SKP1 connects cell cycle regulators to the ubiquitin proteolysis machinery through a novel motif, the F-box, Cell 86 (1996), 263-274.

[20] H. Chen, I. Thalmann and J.C. Adams et al., cDNA cloning, tissue distribution, and chromosomal localization of Ocp2, a gene encoding a putative transcription-associated factor predominantly expressed in the auditory organs, Genomics $\mathbf{2 7}$ (1995), 389-398.

[21] E.R. Yoho, G.N. Thomopoulos, I. Thalmann, R. Thalmann and B.A. Schulte, Localization of organ of Corti protein II in the adult and developing gerbil cochlea, Hear Res 104 (1997), $47-56$.

[22] T.M. Henzl, J. O'Neal, R. Killick, I. Thalmann and R. Thalmann, OCP1, an F-box protein, colocalizes with OCP2/SKP1 in the cochlear epithelial gap-junction region, Hear Res (2001), in press

[23] J.E. Saffitz, J.G. Laing and K.A. Yamada, Connexin expression and turnover: Implications for cardiac excitability, Circ Res 86 (2000), 723-728.
[24] J. Edwards, N. Marziano and D. Becker et al., Co-localisation of connexin b2 [26] and connexin b6 [30] in cochlear gap junctions, Abstr Assoc Res Otolaryngol (2001), 150.

[25] J.H. Xia, C.Y. Liu and B.S. Tang et al., Mutations in the gene encoding gap junction protein beta-3 associated with autosomal dominant hearing impairment, Nat Genet 20 (1998), 370-373.

26] M. Senarita, I. Thalmann, O. Shibasaki and R. Thalmann, Calcium-binding proteins in organ of Corti and basilar papilla: CBP-15, an unidentified calcium-binding protein of the inner ear, Hear Res 90 (1995), 169-175.

27] I. Thalmann, O. Shibasaki, T.H. Comegys, M.T. Henzl, M. Senarita and R. Thalmann, Detection of a beta-parvalbumin isoform in the mammalian inner ear, Biochem Biophys Res Commun 215 (1995), 142-147.

[28] N. Sakaguchi, M. Henzl, I. Thalmann, R. Thalmann and B.A. Schulte, Oncomodulin is expressed exclusively by outer hair cells in the organ of Corti, J Histochem Cytochem 46 (1998), 29-39.

[29] I. Thalmann, R. Thalmann and M.T. Henzl, Novel calcium sensor in the outer hair cells: Quantitation of oncomodulin. Primary Sensory Neuron, Highlights in Hair Cell Biology 2 (1998), 283-296

[30] J.P. MacManus, Occurrence of a low molecular weight calcium-binding protein in neoplastic liver, Cancer Res 39 (1979), 3000-3005.

[31] T.S. Sridhar, M.C. Liberman, M.C. Brown and W.F. Sewell, A novel cholinergic 'slow effect' of olivocochlear stimulation on cochlear potentials in the guinea pig, J Neurosci 15 (1995), 3667-3678.

[32] A.N. Salt, Fluid homeostasis in the inner ear, in: Meniere's disease, J.P. Harris, ed.. Kugler, The Hague, 1999, pp. 93-101.

[33] H. Rask-Andersen, J.E. DeMott, D. Bagger-Sjöbäck and A.N. Salt, Morphological changes of the endolymphatic sac induced by microinjection of artificial endolymph into the cochlea, Hear Res 138 (1999), 81-90.

[34] R. Thalmann, Source and role of macromolecules of endolymph, Acta Otolaryngol 119 (1999), 293-296.

[35] G. VanCamp and R. Smith, http://www.uia.ac.be/dnalab/hhh/.

[36] R.W. Baloh, V. Honrubia and K. Jacobson, Benign positional vertigo: Clinical and oculographic features in 240 cases, $\mathrm{Neu}$ rol 37 (1987), 371-378.

[37] Y. Harada, S. Kasuga and N. Mori, The process of otoconia formation in guinea pig utricular supporting cells, Acta Otolaryngol 118 (1998), 74-79.

[38] Y. Wang, P. Kowalski, I. Thalmann, D.M. Ornitz, D.L. Mager and R. Thalmann, Otoconin-90, the mammalian otoconial matrix protein contains two domains of homology to secretory phospholipase A2, Proc Natl Acad Sci (USA) 95 (1998), $15345-15350$.

[39] R. Thalmann, E. Ignatova, B. Kachar, D.M. Ornitz and I. Thalmann, in: The vestibular system in health and disease, J.A. Goebel and S. Highstein, eds, 2001, in press.

[40] I. Thalmann, T.H. Comegys, S.Z. Liu, Z. Ito and R. Thalmann, Protein profiles of perilymph and endolymph of the guinea pig, Hear Res 63 (1992), 37-42.

[41] I. Thalmann and J.G. Neely, Progressive hearing loss and the perilymphatic fistula, Seminars in Hearing 16 (1995), 214 219.

[42] L.P. Gartner and J.L. Hiatt, Color textbook of histology, Saunders, Philadelphia, 1997, pp. 436.

[43] A.J. Vander, J.H. Sherman and D.S. Luciano, Human Physiology, New York-St. Louis, McGraw Hill, 1990, pp. 245. 
[44] N.B. Slepecky, Structure of the mammalian cochlea, in: The cochlea, P. Dallos, A.N. Popper and R.R. Fay, eds, Springer, New York, 1996, pp. 46

[45] S. Hatakeyama, M. Kitagawa and K. Nakayama et al., Ubiquitin-dependent degradation of $\mathrm{I} \kappa \mathrm{B} \alpha$ is mediated by a ubiquitin ligase Skp1/Cul 1/F-box protein FWD1, Proc Natl Acad Sci 96 (1999), 3859-3863.

[46] H.F. Schuhknecht, Pathology of the ear, Harvard University Press, Cambridge, MA, 1974, pp. 454. 


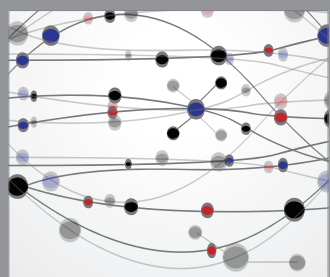

The Scientific World Journal
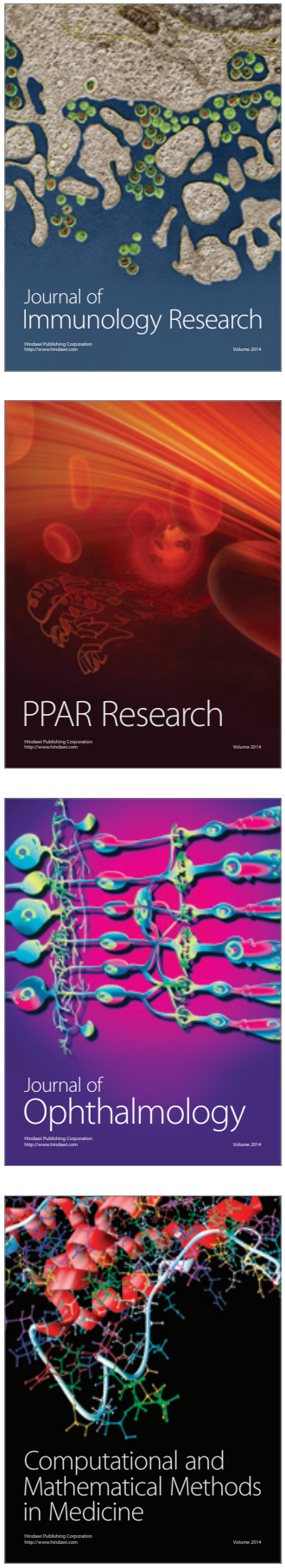

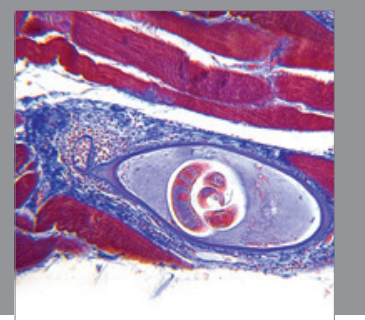

Gastroenterology

Research and Practice
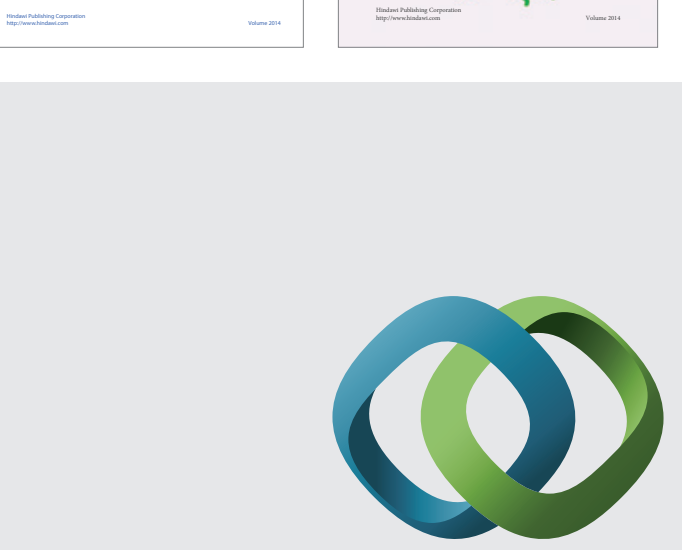

\section{Hindawi}

Submit your manuscripts at

http://www.hindawi.com
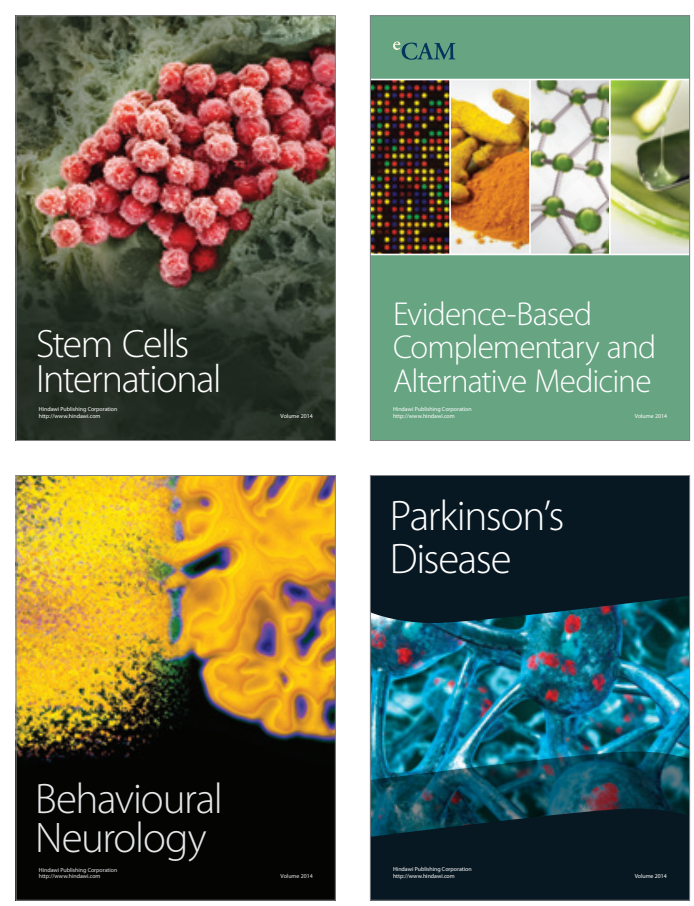

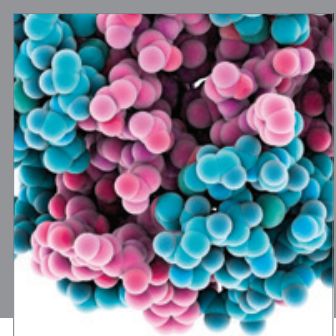

Journal of
Diabetes Research

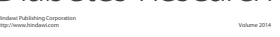

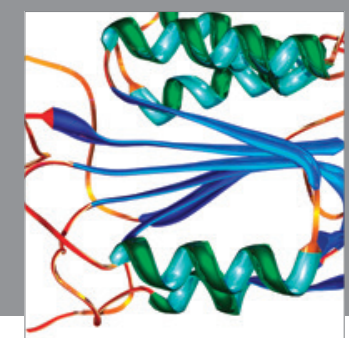

Disease Markers
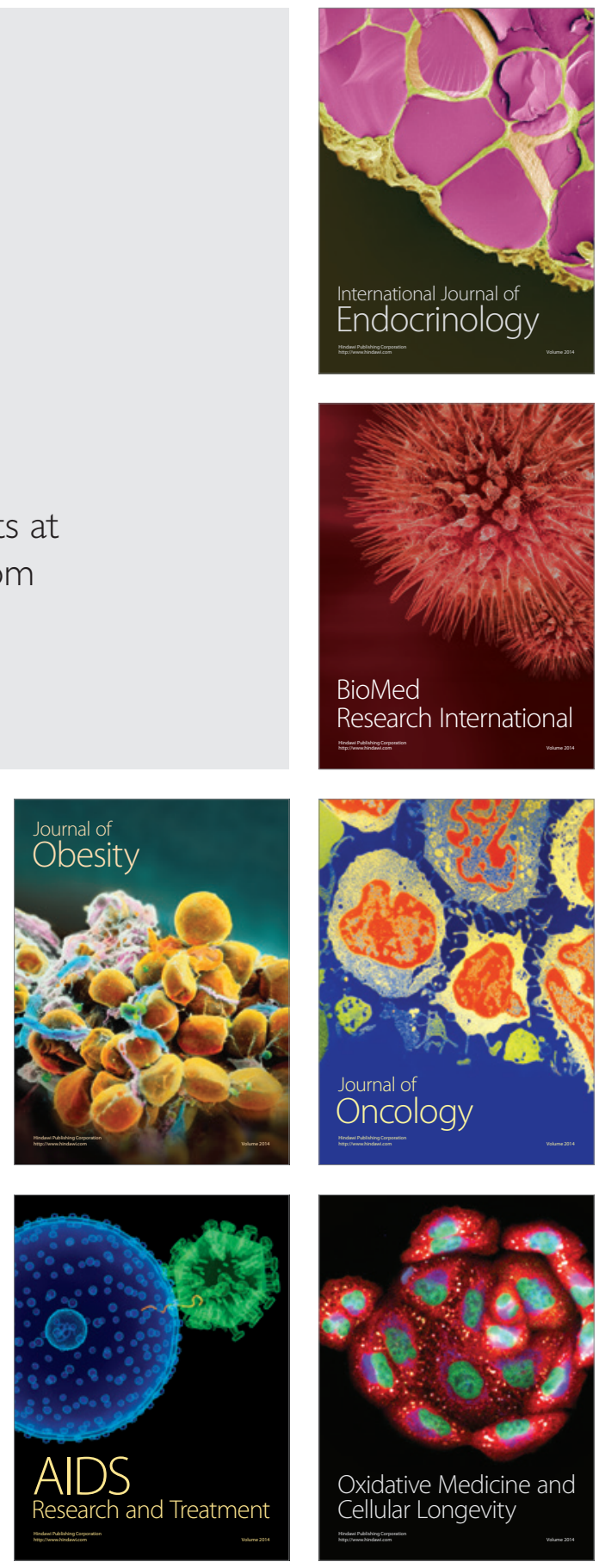